\title{
Modelling the use of impulsive vaccination to control Rift Valley Fever virus transmission
}

\section{Chen-Xia Yang and Lin-Fei Nie*}

"Correspondence: Ifnie@163.com College of Mathematics and System Sciences, Xinjiang University,

Urumqi, 830046, P.R. China

\begin{abstract}
In this paper, we propose a vector-bone dynamical model to against the transmission of Rift Valley Fever (RVF) between ruminants and mosquitoes, where impulsive vaccination for susceptible ruminants is introduced. By using the comparison principle, integral and differential inequalities, and some of analytical skills, the threshold values for the stability of the disease-free periodic solution and uniform persistence of disease are obtained. These values characterize the evolution and extinction of the disease. Numerical simulations are carried out to illustrate the main theoretical results and the feasibility of the impulsive vaccination control strategy.
\end{abstract}

MSC: 34A37; 34D23; 92D30

Keywords: Rift Valley Fever; impulsive vaccination; extinction; asymptotical stability; uniform persistence

\section{Introduction}

Infectious diseases have great impact on human and animals life, which are always aroused by viruses, parasites, bacteria, fungi, and some microorganisms with pathogen. These diseases spread to human (or animals) with directly or indirectly ways. There are significant data showing that many infectious diseases spread with mediums, such as Measles, Dengue fever, Plague, Hydrophobia, and so on. Rift Valley Fever virus (RVFV) is an important mosquito-borne viral zoonsis in North Africa and Kenya, which is a member of the Phlebovirus genus in the Bunyaviridae family. RVFV was first discovered in Kenya in the early 1930s [1]. RVFV is spread by either touching infected animal blood, breathing in the air around an infected animal being butchered, drinking raw milk from an infected animal, or the bite of infected mosquitoes. Animals such as cows, sheep, goats, and camels may be affected $[2,3]$.

Using mathematical models to investigate the transmission rules of RVFV disease is beneficial to control diseases in medical science, so the dynamic behaviors of the diseases are investigated in many literatures. We refer to some of them, [4-8] and the references therein. Particularly, Gaff et al. [9] investigated an epidemiological model for RVFV and found the stability of the disease-free equilibrium. Saul et al. [10] investigated the dynamical behavior of RVFV with human host and computed the disease threshold $\mathcal{R}_{0}$ for ana-

(c) 2016 Yang and Nie. This article is distributed under the terms of the Creative Commons Attribution 4.0 International License (http://creativecommons.org/licenses/by/4.0/), which permits unrestricted use, distribution, and reproduction in any medium, provided you give appropriate credit to the original author(s) and the source, provide a link to the Creative Commons license, and indicate if changes were made. 
lyzing the local stability of equilibria. Xue et al. [11] presented a new compartmentalized model for RVFV and used ordinary differential equations to assess disease spread in both time and space. With the latter driven as a function of contact networks, Gao et al. [12] investigated the spatial spread of RVFV and proposed a three-patch model, introduced the basic reproduction number for each patch, and then established the threshold dynamics of the model. Of course, mathematical models also used to discuss the transmission rules of other vector-borne disease [13-15] and the references therein.

Recently, vaccines are generally used to protect animals from Rift Valley Fever in endemic regions. Right now, two types of vaccines are available for susceptible animals to reduce amplification of the virus: inactivated whole-virus and live-attenuated Smithburn vaccines [16]. Inactivated vaccines can be applied to ruminants of all ages without causing abortions, but they are expensive, and repeated doses are required. In comparison, live-attenuated vaccines are cheap and effective since they confer a lifelong immunity with a single dose. But the undesirable side effects are obvious: they may lead to fetal abnormalities and abortions in pregnant ruminants, and there is the safety concern of reversion to virulence [17]. Based on this, Farida et al. [18] used the mathematical model to investigate RVFV among ruminants and considered continuous vaccination for animals and found that vaccination was efficient to reduce the loss of animals. We know, however, that the continuous vaccination is not practical in the reality living, and the control measures are only employed in the particular moment. So for this reason, infectious models with impulsive vaccination are concerned by more and more scholars. For example, Sabin [19] controlled successfully measles and poliomyelitis throughout Central and South America, and another example of successful application of this strategy is the U.K. vaccination action against measles in [20]. Using a SIR epidemic model, Shulgin et al. [21] showed that under a planned pulse vaccination region, the sate of model converges to a stable sate with which the size of infectious population is zero. This result shows that the pulse vaccination may lead to the eradication of infectious disease, provided that the magnitude of vaccination keeps a rational proportion and the period of pulses is sustained. d'Onofrio [22] proposed a SEIR epidemic model based pulse vaccination strategy by which the local and global asymptotic stabilities of the periodic eradication solution are analyzed. More related research works on the dynamical behaviors of epidemic models with pulse vaccination also can be found in [23-27] and the references therein.

Looking at the results of existing researches on epidemic dynamical models with impulsive vaccination and using mathematical model to investigate vector-borne epidemic model, the dynamic behavior with impulsive vaccination strategy is not investigated. Therefore, we propose a novel dynamical model to control the transmission of RVFV, where impulsive vaccinate for susceptible ruminant at regular interval is proposed. The main purpose is to investigate the impulsive vaccination control strategy that governs whether the disease dies out or not, and further to examine how the control strategy affects the prevention and control of RVFV disease. The organization of this paper is as follows. We present preliminaries and formulate the control model of RVF with impulsive vaccination in the next section. In Section 3, we consider the global stability of the free-disease periodic solution and give a threshold value for the disease-eliminating. In Section 4, we 
discuss the uniform persistence of the disease. Numerical simulation and discussion are carried in Section 5.

\section{Model formulation and preliminaries}

The traditional RVFV model is to divide ruminant population into three classes, the susceptible $S$, infectious $I$, and recovered $R$, and female mosquitoes are divided into two classes, uninfected $U$ and infectious $V$. In order to formulate the control model, we have the following assumptions.

$\left(A_{1}\right)$ The ruminant population is recruited with rate $\Lambda, \mu$ is the natural death rate (including slaughter) of ruminant, with RVFV owing to infection with RVFV also causing mortality in ruminants. We assume that ruminants die due to RVFV infection at rate $d$ (including slaughter) and do not consider infection with RVFV causing abortion in ruminant.

$\left(A_{2}\right)$ An infectious mosquito bites ruminant, and ruminant is infected by infectious mosquito successfully by rate $p_{r}$; in turn, an infectious mosquito bites infectious ruminant and is infected by infectious ruminant successfully with rate $p_{m}$, and we assume that each female mosquito bites at a constant rate $a$.

$\left(A_{3}\right)$ Assume that $\xi$ is the growth rate of mosquitoes and $M_{0}$ is the capacity for mosquitoes. Infection with RVFV induces life-long immunity in ruminants at a rate of $\gamma$, and we also assume that $\eta$ is the natural birth/death rate of mosquitoes. Moreover, in this paper, we do not take into account vertical transmission of RVFV in mosquitoes since vertical transmission is rare.

$\left(A_{4}\right)$ With the consideration of mechanism of prevention and control for the spread of RVFV, we consider impulsive vaccination, and only susceptible ruminants are vaccinated at rate $\phi$. Assuming that after successful vaccination, ruminants are completely immune to the virus and move to the recovery compartment with immunity.

Under aforementioned assumptions, we come to the control model that is governed by the following ordinary differential equation with impulsive vaccination:

- $t \neq n T, n=1,2, \ldots$

$\left\{\begin{array}{l}\frac{\mathrm{d} S(t)}{\mathrm{d} t}=\Lambda-a p_{r} S(t) V(t)-\mu S(t), \\ \frac{\mathrm{d}(t)}{\mathrm{d} t}=a p_{r} S(t) V(t)-(\mu+d+\gamma) I(t), \\ \frac{\mathrm{d} R(t)}{\mathrm{d} t}=\gamma I(t)-\mu R(t), \\ \frac{\mathrm{d} U(t)}{\mathrm{d} t}=\xi(U(t)+V(t))-\frac{\xi-\eta}{M_{0}}(U(t)+V(t))^{2}-a p_{m} U(t) I(t)-\eta U(t), \\ \frac{\mathrm{d} V(t)}{\mathrm{d} t}=a p_{m} U(t) I(t)-\eta V(t) .\end{array}\right.$

- $t=n T, n=1,2, \ldots$

$\left\{\begin{array}{l}S\left(t^{+}\right)=(1-\phi) S(t) \\ I\left(t^{+}\right)=I(t) \\ R\left(t^{+}\right)=R(t)+\phi S(t) \\ U\left(t^{+}\right)=U(t) \\ V\left(t^{+}\right)=V(t)\end{array}\right.$ 
The initial condition of models (1) and (2) is given as $\left(S_{0}, I_{0}, R_{0}, U_{0}, V_{0}\right) \in \mathbb{R}_{+}^{5}=\left\{\left(x_{1}, x_{2}, x_{3}\right.\right.$, $\left.\left.x_{4}, x_{5}\right): x_{i} \geq 0, i=1,2,3,4,5\right\}$.

The total number of mosquitoes at time $t$ is denoted by $M(t)$, where $M(t)=U(t)+V(t)$. From the equations of mosquitoes of models (1) and (2) we have

$$
\frac{\mathrm{d} M(t)}{\mathrm{d} t}=(\xi-\eta) M(t)-\frac{\xi-\eta}{M_{0}}(M(t))^{2} .
$$

Obviously, by the preceding, we have that $M(t)$ converges to $M_{0}$ as $t \rightarrow \infty$ for any positive initial value. Therefore, we can consider the following limit system of models (1) and (2):

$$
\left\{\begin{array}{l}
\frac{\mathrm{d} S(t)}{\mathrm{d} t}=\Lambda-a p_{r} S(t) V(t)-\mu S(t), \\
\frac{\mathrm{d} I(t)}{\mathrm{d} t}=a p_{r} S(t) V(t)-(\mu+d+\gamma) I(t), \\
\frac{\mathrm{d} R(t)}{\mathrm{d} t}=\gamma I(t)-\mu R(t), \\
\frac{\mathrm{d} U(t)}{\mathrm{d} t}=\eta M_{0}-a p_{m} U(t) I(t)-\eta U(t), \\
\frac{\mathrm{d} V(t)}{\mathrm{d} t}=a p_{m} U(t) I(t)-\eta V(t), \\
\left.\begin{array}{l}
S\left(t^{+}\right)=(1-\phi) S(t), \\
I\left(t^{+}\right)=I(t), \\
R\left(t^{+}\right)=R(t)+\phi S(t), \\
U\left(t^{+}\right)=U(t), \\
V\left(t^{+}\right)=V(t)
\end{array}\right\} \quad t=n T, n=1,2, \ldots . \\
t
\end{array}\right\}
$$

From biological considerations, we analyze the dynamical behaviors of model (3) in the closed set

$$
\Omega=\left\{(S, I, R, U, V) \in \mathbb{R}_{+}^{5}: 0 \leq S(t)+I(t)+R(t) \leq \frac{\Lambda}{\mu}, 0 \leq U(t)+V(t) \leq M_{0}\right\} .
$$

On the nonnegative of solutions for model (3) we have the following lemma.

Lemma 1 Each component of any solution of model (3) is nonnegative, and $\Omega$ is a positively invariant.

The proof of Lemma 1 is obvious, and hence we omit it here.

Next, we consider the general impulsive differential equation

$$
\begin{cases}\frac{\mathrm{d} z(t)}{\mathrm{d} t}=a-b z(t), & t \neq n T \\ z\left(t^{+}\right)=(1-p) z(t), & t=n T, n=1,2, \ldots\end{cases}
$$

where $a>0, b>0$, and $0<p<1$.

The following Lemma 2 is on the existent and stability of positive periodic solution for equation (4). Though its proof is straightforward, but useful. 
Lemma 2 Equation (4) has a unique positive periodic solution

$$
z_{p}(t)=\frac{a}{b}+\left(z^{*}-\frac{a}{b}\right) \exp \{-b(t-n T)\}, \quad n T<t \leq(n+1) T,
$$

where

$$
z^{*}=\frac{a(1-p)(1-\exp \{-b T\})}{b[1-(1-p) \exp \{-b T\}]}
$$

and $z_{p}(t)$ is globally asymptotically stable.

Finally, for the convenience of further statements, we introduce the definition on the uniform persistence of disease.

Definition 1 The disease in model (3) is said to be uniform persistent if there exists a positive constant $m^{*}$ such that $\liminf _{t \rightarrow \infty} I(t) \geq m^{*}$ and $\liminf _{t \rightarrow \infty} V(t) \geq m^{*}$.

\section{The existence and stability of the disease-free periodic solution}

In this section, we discuss the existence and stability of the disease-free periodic solution of model (3). Supposing that the infectious individuals are completely absent, that is, $I(t)=$ $V(t)=0$, model (3) becomes of the following style:

$$
\left\{\begin{array}{l}
\frac{\mathrm{d} S(t)}{\mathrm{d} t}=\Lambda-\mu S(t), \\
\frac{\mathrm{d} R(t)}{\mathrm{d} t}=-\mu R(t), \\
\frac{\mathrm{d} U(t)}{\mathrm{d} t}=\eta M_{0}-\eta U(t), \\
S\left(t^{+}\right)=(1-\phi) S(t), \\
R\left(t^{+}\right)=R(t)+\phi S(t), \\
U\left(t^{+}\right)=U(t)
\end{array}\right\} \quad t \neq n T,
$$

From the first and fourth equations of model (5) we have

$$
\begin{cases}\frac{\mathrm{d} S(t)}{\mathrm{d} t}=\Lambda-\mu S(t), & t \neq n T, \\ S\left(t^{+}\right)=(1-\phi) S(t), & t=n T, n=1,2, \ldots\end{cases}
$$

In view of Lemma 2, it follows that model (6) has a unique positive periodic solution

$$
S_{p}(t)=\frac{\Lambda}{\mu}+\left(S^{*}-\frac{\Lambda}{\mu}\right) \exp \{-\mu(t-n T)\}, \quad n T<t \leq(n+1) T
$$

where

$$
S^{*}=\frac{\Lambda(1-\phi)(1-\exp \{-\mu T\})}{\mu[1-(1-\phi) \exp \{-\mu T\}]}
$$

which is globally asymptotically stable. Let $N(t)=S(t)+R(t)$. From model (5) we have $\mathrm{d} N(t) / \mathrm{d} t=\Lambda-\mu N(t)$ and $\lim _{t \rightarrow \infty} N(t)=\Lambda / \mu$. Hence, $\lim _{t \rightarrow \infty} R(t)=\Lambda / \mu-\lim _{t \rightarrow \infty} S(t)$, and we denote $R_{p}(t)=\Lambda / \mu-S_{p}(t)$. 
Further, from the third and sixth equations of model (5) we easily get that

$$
\lim _{t \rightarrow \infty} U(t)=M_{0}:=U_{p}(t)
$$

Moreover, from the above discussion we have that model (5) has a globally asymptotically stable periodic solution $\left(S_{p}(t), R_{p}(t), U_{p}(t)\right)$.

Let

$$
\mathcal{R}_{0}=\frac{1}{\theta^{*} T} \int_{0}^{T}\left[a p_{r} S_{p}(t)+a p_{m} U_{p}(t)\right] \mathrm{d} t
$$

where $\theta^{*}=\min \{\mu+d+\gamma, \eta\}$. On the global asymptotic stability of a disease-free periodic solution of model (3) we have the following theorem.

Theorem 1 If $\mathcal{R}_{0}<1$, then model (3) has a unique disease-free periodic solution $\left(S_{p}(t), 0\right.$, $\left.R_{p}(t), U_{p}(t), 0\right)$, which is globally asymptotically stable.

Proof Because $\mathcal{R}_{0}<1$, we can chose $\varepsilon>0$ small enough such that

$$
\int_{0}^{T}\left[a p_{r} S_{p}(t)+a p_{m} U_{p}(t)+\left(a p_{r}+a p_{m}\right) \varepsilon-\theta^{*}\right] \mathrm{d} t<0 .
$$

From the first equation of model (3) it follows that

$$
\begin{cases}\frac{\mathrm{d} S(t)}{\mathrm{d} t}=\Lambda-a p_{r} S(t) V(t)-\mu S(t) \leq \Lambda-\mu S(t), & t \neq n T, \\ S\left(t^{+}\right)=(1-\phi) S(t), & t=n T, n=1,2, \ldots\end{cases}
$$

Now, we consider the following comparison impulsive differential equation:

$$
\begin{cases}\frac{\mathrm{d} \widehat{S}(t)}{\mathrm{d} t}=\Lambda-\mu \widehat{S}(t), & t \neq n T \\ \widehat{S}\left(t^{+}\right)=(1-\phi) \widehat{S}(t), & t=n T, n=1,2, \ldots\end{cases}
$$

By Lemma 2, model (13) has a unique positive periodic solution given by (7) and (8). Let $(S(t), I(t), R(t), U(t), V(t))$ be a solution of model (3) with $S\left(0^{+}\right)=S_{0}>0$, and $\widehat{S}(t)$ be a solution of model (13) with $\widehat{S}\left(0^{+}\right)=S\left(0^{+}\right)$. Therefore, by the comparison theorem of impulsive differential equation (for more details, see $[28,29]$ ) and Lemma 2 there is an integer $n_{1}>0$ such that, for all $n \geq n_{1}$,

$$
S(t) \leq \widehat{S}(t)<S_{p}(t)+\varepsilon, \quad t \in(n T,(n+1) T]
$$

Further, from the fourth and ninth equations of model (3) we have

$$
\frac{\mathrm{d} U(t)}{\mathrm{d} t} \leq \eta M_{0}-\eta U(t)
$$

This, together with the comparison theorem of ordinary differential equation, shows that there exists an integer $n_{2} \geq n_{1}$ such that

$$
U(t) \leq U_{p}(t)+\varepsilon, \quad t \in(n T,(n+1) T], n \geq n_{2} .
$$


Then, from inequalities (14) and (15) and from the second and fifth equations of model (3) we get

$$
\begin{aligned}
\frac{\mathrm{d}(I(t)+V(t))}{\mathrm{d} t} & =a p_{r} S(t) V(t)-(\mu+d+\gamma) I(t)+a p_{m} U(t) I(t)-\eta V(t) \\
& \leq a p_{r} S(t)(I(t)+V(t))+a p_{m} U(t)(I(t)+V(t))-\theta(I(t)+V(t)) \\
& \leq\left[a p_{r} S_{p}(t)+a p_{m} U_{p}(t)+\left(a p_{r}+a p_{m}\right) \varepsilon-\theta^{*}\right](I(t)+V(t))
\end{aligned}
$$

for all $t \geq n T$ and $n \geq n_{2}$. By (11) we can get $\lim _{t \rightarrow \infty}(I(t)+V(t))=0$, that is,

$$
\lim _{t \rightarrow \infty} I(t)=\lim _{t \rightarrow \infty} V(t)=0
$$

since $I(t) \geq 0$ and $V(t) \geq 0$. Therefore, there is an integer $n_{3} \geq n_{2}$ such that

$$
0<I(t), \quad V(t)<\varepsilon_{0}:=\min \left\{\frac{\varepsilon}{2}, \frac{\varepsilon}{2}\left(\frac{a p_{r} \Lambda}{\mu}+\frac{a p_{r} \Lambda(1-\phi)\left(1-e^{-\mu T}\right)}{\mu\left[1-(1-\phi) e^{-\mu T}\right]}-\frac{a p_{r} \Lambda}{\mu} e^{-\mu T}\right)^{-1}\right\}
$$

for all $t \geq n_{3} T$. From the above and the first equation of model (3) we have

$$
\frac{\mathrm{d} S(t)}{\mathrm{d} t}=\Lambda-a p_{r} S(t) V(t)-\mu S(t) \geq \Lambda-a p_{r} \frac{\Lambda}{\mu} \varepsilon_{0}-\mu S(t) \quad \text { for all } t \geq n_{3} T .
$$

Consider the following comparison impulsive differential equation:

$$
\begin{cases}\frac{\mathrm{d} x(t)}{\mathrm{d} t}=\Lambda-a p_{r} \frac{\Lambda}{\mu} \varepsilon_{0}-\mu x(t), & t \neq n T, \\ x\left(t^{+}\right)=(1-\phi) x(t), & t=n T, n=1,2, \ldots\end{cases}
$$

By Lemma 2, model (18) has a unique positive periodic solution $x_{p}(t)$, which is globally asymptotically stable,

$$
x_{p}(t)=\frac{\Lambda-\frac{a p_{r} \Lambda \varepsilon_{0}}{\mu}}{\mu}+\left(x^{*}-\frac{\Lambda-\frac{a p_{r} \Lambda \varepsilon_{0}}{\mu}}{\mu}\right) \exp \{-\mu(t-n T)\}, \quad n T<t \leq(n+1) T
$$

with

$$
x^{*}=\frac{\left(\Lambda-\frac{a p_{r} \Lambda \varepsilon_{0}}{\mu}\right)(1-\phi)(1-\exp \{-\mu T\})}{\mu[1-(1-\phi) \exp \{-\mu T\}]} .
$$

Hence, by (17) and the comparison theorem of impulsive differential equation, there exists an integer $n_{4} \geq n_{3}$ such that

$$
S(t)>x_{p}(t)-\frac{\varepsilon}{2}, \quad n T<t \leq(n+1) T .
$$

Using the comparison theorem and the expressions of $\varepsilon_{0}$ and $S_{p}$, we obtain that there is an integer $n_{5} \geq n_{4}$ such that, for $n \geq n_{5}$,

$$
x_{p}(t) \geq S_{p}(t)-\frac{\varepsilon}{2}, \quad n T<t \leq(n+1) T .
$$


On the other hand, from the fourth and ninth equations of model (3) we get

$$
\frac{\mathrm{d} U(t)}{\mathrm{d} t}=\eta M_{0}-a p_{m} U(t) I(t)-\eta U(t) \geq \eta M_{0}-a p_{m} M_{0} \varepsilon_{0}-\eta U(t) .
$$

Similarly to the above discussion, there exists an integer $n_{6} \geq n_{5}$ such that, for $t \geq n_{6} T$,

$$
U(t) \geq M_{0}-\frac{a p_{m} M_{0}}{\eta} \varepsilon_{0}-\varepsilon
$$

Since $\varepsilon$ is small enough, it follows that

$$
\lim _{t \rightarrow \infty} U(t)=U_{p}(t)
$$

where we also used the fact (15). Further, by (14), (19), and (20) we have

$$
\lim _{t \rightarrow \infty} S(t)=S_{p}(t)
$$

Thus,

$$
\lim _{t \rightarrow \infty} R(t)=\lim _{t \rightarrow \infty}(N(t)-S(t)-I(t))=\frac{\Lambda}{\mu}-S_{p}(t)=R_{p}(t) .
$$

By (16) and (21)-(23) we know the disease-free periodic solution $\left(S_{p}(t), 0, R_{p}(t), U_{p}(t), 0\right)$ of model (3) is globally asymptotically stable if $\mathcal{R}_{0}<1$. This completes the proof.

Next, we consider the critical vaccination proportion, that is, the value $\phi=\phi^{*}$ such that $\mathcal{R}_{0}\left(\phi^{*}\right)=1$, where $\mathcal{R}_{0}\left(\phi^{*}\right)$ is the value for $\mathcal{R}_{0}$ in which $\phi$ is replaced by $\phi^{*}$. We easily calculate that

$$
\phi^{*}=\frac{T \mu\left(\frac{a p_{m} M_{0}}{\theta^{*}}+\frac{a p_{r} \Lambda}{\mu \theta^{*}}-1\right)}{\frac{a p_{r} \Lambda}{\mu \theta^{*}}-\frac{T \mu\left(\frac{a p_{m} M_{0}}{\theta^{*}}+\frac{a p_{r} \Lambda}{\mu \theta^{*}}-1\right)}{1-\exp \{-\mu T\}} \exp \{-\mu T\}} .
$$

Further, from (10) we have

$$
\frac{\mathrm{d} \mathcal{R}_{0}}{\mathrm{~d} \phi}=\frac{-a p_{r} \Lambda(1-\exp \{-\mu T\})^{2}}{\mu^{2} \theta^{*} T[1-(1-\phi) \exp \{-\mu T\}]^{2}}<0 .
$$

Therefore, $\mathcal{R}_{0}<1$ only when $\phi>\phi^{*}$. We have the following theorem, which is a direct consequence of Theorem 1 .

Theorem 2 The disease-free periodic solution $\left(S_{p}(t), 0, R_{p}(t), U_{p}(t), 0\right)$ of model (3) is globally asymptotically stable when $\phi>\phi^{*}$, where $\phi^{*}$ is given by (24).

\section{The uniform persistence of RVFV}

In this section, we discuss the uniform persistence of RVFV for model (3). For any $\tilde{p}>0$ and $t>0$, we define

$$
H(\tilde{p}, t)=a p_{r} \tilde{p} S_{p}(t)+\eta-\frac{1}{\tilde{p}} a p_{m} U_{p}(t)-(\mu+d+\gamma)
$$


and

$$
F(\tilde{p}, t)=\tilde{p} I(t)-V(t)
$$

where $S_{p}(t)$ and $U_{p}(t)$ are given by (7) and (9), respectively, and $I(t)$ and $V(t)$ are elements of solutions of model (3).

The following theorem is on the uniform persistence of disease.

Theorem 3 If there exists positive constants $\tilde{p}$ and $\alpha$ such that

$$
\begin{aligned}
& \mathcal{R}_{1}=\int_{0}^{T}\left[a p_{r} \tilde{p} S_{p}(\tau)-(\mu+d+\gamma)\right] \mathrm{d} \tau>0, \\
& \mathcal{R}_{2}=\int_{0}^{T}\left[\frac{a p_{m}}{\tilde{p}} U_{p}(\tau)-\eta\right] \mathrm{d} \tau>0,
\end{aligned}
$$

and $H(\tilde{p}, t)<0$ for all $t \geq 0$, then the Rift Valley Fever disease in model (3) is uniformly persistent.

Proof Since $H(\tilde{p}, t)<0$, we can chose a positive constant $\alpha$ such that $H(\tilde{p}, t)<-\alpha$, and by (25) and (26) we can choose small enough positive constants $\varepsilon, \varepsilon_{1}, \eta_{1}, \eta_{2}$, and $\eta_{3}$ such that

$$
\begin{aligned}
& \int_{0}^{T}\left[\frac{a p_{m}}{\tilde{p}}\left(U_{p}(\tau)-\varepsilon\right)-\eta\right] \mathrm{d} \tau>\eta_{1}, \\
& \int_{0}^{T}\left[a p_{r} \tilde{p}\left(S_{p}(\tau)-\varepsilon\right)-(\mu+d+\gamma)\right] \mathrm{d} \tau>\eta_{2}, \\
& \int_{0}^{T}\left[a p_{m}\left(U_{p}(\tau)+\varepsilon\right) \varepsilon-\eta \varepsilon_{1}\right] \mathrm{d} \tau<-\eta_{3},
\end{aligned}
$$

and

$$
a p_{r} \tilde{p}\left(S_{p}(t)+\varepsilon\right)+\eta-(\mu+d+\gamma)-\frac{a p_{m}}{\tilde{p}}\left(U_{p}(t)-\varepsilon\right)<0 .
$$

First, we prove that

$$
\limsup _{t \rightarrow \infty} I(t) \geq \varepsilon
$$

If (31) were not true, then there would exist a positive integer $n_{1}$ such that $0<I(t)<\varepsilon$ for all $t \geq n_{1} T$.

If $V(t) \geq \varepsilon_{1}$ for all $t \geq n_{1} T$, then from the fifth equation of model (3) and the fact $0<$ $I(t)<\varepsilon$ we have

$$
\begin{aligned}
V(t) & =V\left(n_{1} T\right)+\int_{n_{1} T}^{t}\left[a p_{m} U(s) I(s)-\eta V(s)\right] \mathrm{d} s \\
& \leq V\left(n_{1} T\right)+\int_{n_{1} T}^{t}\left[a p_{m}\left(U_{p}(s)+\varepsilon\right) \varepsilon-\eta \varepsilon_{1}\right] \mathrm{d} s
\end{aligned}
$$


for all $t \geq n_{1} T$. Thus, from (29) it follows that $\lim _{t \rightarrow \infty} V(t)=-\infty$. This is in contradiction with $V(t)>0$. Therefore, there exists $t_{1} \geq n_{1} T$ such that $V\left(t_{1}\right)<\varepsilon_{1}$. Now, we claim that

$$
V(t)<\varepsilon_{1}+a p_{m}\left(M_{0}+\varepsilon\right) \varepsilon T
$$

for all $t \geq t_{1}$. Otherwise, there is $t_{2}>t_{1}$ such that $V\left(t_{2}\right)>\varepsilon_{1}+a p_{m}\left(M_{0}+\varepsilon\right) \varepsilon T$. Then there must exist $t_{3} \in\left(t_{1}, t_{2}\right)$ such that $V\left(t_{3}\right)=\varepsilon_{1}$ and $V(t)>\varepsilon_{1}$ for $t \in\left(t_{3}, t_{2}\right)$. We can chose a positive integer $m$ such that $t_{2} \in\left(t_{3}+m T, t_{3}+(m+1) T\right)$. Integrating the fifth equation of model (3) from $t_{3}$ to $t_{2}$, we get

$$
\begin{aligned}
\varepsilon_{1}+a p_{m}\left(M_{0}+\varepsilon\right) \varepsilon T & <V\left(t_{2}\right)=V\left(t_{3}\right)+\int_{t_{3}}^{\tau_{2}}\left[a p_{m} U(s) I(s)-\eta V(s)\right] \mathrm{d} s \\
& <\varepsilon_{1}+\left(\int_{t_{3}}^{t_{3}+m T}+\int_{t_{3}+m T}^{t_{2}}\right)\left[a p_{m}\left(U_{p}(s)+\varepsilon\right) \varepsilon-\eta \varepsilon_{1}\right] \mathrm{d} s \\
& <\varepsilon_{1}+a p_{m}\left(M_{0}+\varepsilon\right) \varepsilon T,
\end{aligned}
$$

where we also used (29). This is a contradiction. Thus, (32) is valid.

From the first equation of model (3),we have

$$
\frac{\mathrm{d} S(t)}{\mathrm{d} t}=\Lambda-a p_{r} S(t) V(t)-\mu S(t) \geq \Lambda-a p_{r} \frac{\Lambda}{\mu}\left[\varepsilon_{1}+a p_{m}\left(M_{0}+\varepsilon\right) \varepsilon T\right]-\mu S(t) .
$$

Now, we consider the following comparison impulsive differential equation:

$$
\begin{cases}\frac{\mathrm{d} \widetilde{S}(t)}{\mathrm{d} t}=\Lambda-a p_{r} \frac{\Lambda}{\mu}\left[\varepsilon_{1}+a p_{m}\left(M_{0}+\varepsilon\right) \varepsilon T\right]-\mu \widetilde{S}(t), & t \neq n T, \\ \widetilde{S}\left(t^{+}\right)=(1-\phi) \widetilde{S}(t), & t=n T, n=1,2, \ldots\end{cases}
$$

By Lemma 2, model (34) has a unique positive periodic solution $\widetilde{S}_{p}(t)$, which is globally asymptotically stable,

$$
\begin{aligned}
\widetilde{S}_{p}(t)= & \frac{\Lambda \mu-a p_{r} \Lambda\left[\varepsilon_{1}+a p_{m}\left(M_{0}+\varepsilon\right) \varepsilon T\right]}{\mu^{2}} \\
& +\left(\widetilde{S}_{p}^{*}-\frac{\Lambda \mu-a p_{r} \Lambda\left[\varepsilon_{1}+a p_{m}\left(M_{0}+\varepsilon\right) \varepsilon T\right]}{\mu^{2}}\right) \exp \{-\mu(t-n T)\}
\end{aligned}
$$

for all $n T<t \leq(n+1) T, n=1,2, \ldots$, where

$$
\widetilde{S}_{p}^{*}=\frac{\left[\Lambda \mu-a p_{r} \Lambda\left(\varepsilon_{1}+a p_{m}\left(M_{0}+\varepsilon\right) \varepsilon T\right)\right](1-\phi)(1-\exp \{-\mu T\})}{\mu^{2}[1-(1-\phi) \exp \{-\mu T\}]} .
$$

By (33) and the comparison theorem for impulsive differential equation there exists an integer $n_{2} \geq n_{1}$ such that

$$
S(t)>\widetilde{S}_{p}(t)-\frac{\varepsilon}{2} \quad \text { for all } t \in(n T,(n+1) T], n \geq n_{2} .
$$

Further, by (12) and the comparison theorem for impulsive differential equation there exists an integer $n_{3} \geq n_{2}$ such that

$$
S(t)<S_{p}(t)+\varepsilon
$$


for all $t \in(n T,(n+1) T]$ and $n \geq n_{3}$. Since $\varepsilon$ and $\varepsilon_{1}$ is small enough, from (35) and (36) we have

$$
S_{p}(t)-\varepsilon<S(t)<S_{p}(t)+\varepsilon
$$

On the other hand, from the fourth equation of model (3) we have

$$
\eta M_{0}-a p_{m} M_{0} \varepsilon-\eta U(t)<\frac{\mathrm{d} U(t)}{\mathrm{d} t}=\eta M_{0}-a p_{m} U(t) I(t)-\eta U(t)<\eta M_{0}-\eta U(t)
$$

for $t \geq n_{3} T$. By the comparison theorem of ordinary differential equation,there has an integer $n_{4} \geq n_{3}$ such that

$$
M_{0}-\frac{a p_{m} M_{0}}{\eta} \varepsilon-\varepsilon<U(t)<M_{0}+\varepsilon
$$

for all $t \geq n_{4} T$.

We further prove that there exists a positive integer $n_{5} \geq n_{4}$ such that

$$
F(\tilde{p}, t) \leq 0 \quad \text { for all } t \geq n_{5} T \text {. }
$$

Otherwise, there exist two cases:

(i) $F(\tilde{p}, t)>0$ for all $t \geq n_{4} T$;

(ii) $F(\tilde{p}, t)$ oscillates about 0 for all large $t$.

If case (i) holds, from the fifth equation of model (3) and inequality (38) we have

$$
\frac{\mathrm{d} V(t)}{\mathrm{d} t}>\frac{a p_{m}}{\tilde{p}} U(t) V(t)-\eta V(t)>\left[\frac{a p_{m}}{\tilde{p}}\left(U_{p}(t)-\varepsilon\right)-\eta\right] V(t),
$$

where we used the fact that $F(\tilde{p}, t)>0$. Integrating this inequality from $n_{4} T$ to $t$, we get

$$
V(t)>V(n T) \exp \left\{\int_{n_{4} T}^{t}\left[\frac{a p_{m}}{\tilde{p}}\left(U_{p}(\tau)-\varepsilon\right)-\eta\right] \mathrm{d} \tau\right\} .
$$

From this and from (27) we get that $\lim _{t \rightarrow \infty} V(t)=\infty$. This contradicts with the bound of $V(t)$. So, case (i) is false.

Now, we turn to case (ii). There exist two time sequences $\left\{t_{m}\right\}$ and $\left\{s_{m}\right\}$ satisfying

$$
n_{4} T<s_{1}<t_{1}<s_{2}<t_{2}<\cdots<s_{m}<t_{m}<\cdots
$$

and $\lim _{m \rightarrow \infty} s_{m}=\infty$ such that $F\left(\tilde{p}, s_{m}\right)=0, \mathrm{~d} F\left(\tilde{p}, s_{m}\right) / \mathrm{d} s_{m} \geq 0, F(\tilde{p}, t)>0$ for all $t \in$ $\bigcup_{m=1}^{\infty}\left(s_{m}, t_{m}\right)$, and $F(\tilde{p}, t) \leq 0$ for all $t \notin \bigcup_{m=1}^{\infty}\left(s_{m}, t_{m}\right)$. Therefore, we have $\tilde{p} I\left(s_{m}\right)=V\left(s_{m}\right)$ and

$$
\begin{aligned}
\frac{\mathrm{d} F\left(\tilde{p}, s_{m}\right)}{\mathrm{d} s_{m}} & =\tilde{p} \frac{\mathrm{d} I\left(s_{m}\right)}{\mathrm{d} s_{m}}-\frac{\mathrm{d} V\left(s_{m}\right)}{\mathrm{d} s_{m}} \\
& =V\left(s_{m}\right)\left[a p_{r} \tilde{p} S\left(s_{m}\right)+\eta-(\mu+d+\gamma)-\frac{a p_{m}}{\tilde{p}} U\left(s_{m}\right)\right] \geq 0 .
\end{aligned}
$$


Obviously, this is in contraction with (30). Moreover, by the above discussion (39) is thus valid.

From the second equation of model (3) and from (37) and (39) we have

$$
\frac{\mathrm{d} I(t)}{\mathrm{d} t}=a p_{r} S(t) V(t)-(\mu+d+\gamma) I(t) \geq\left[a p_{r} \tilde{p}\left(S_{p}(t)-\varepsilon\right)-(\mu+d+\gamma)\right] I(t) .
$$

Integrating this inequality from $n_{5} T$ to $t$, it follows that

$$
I(t) \geq I\left(n_{5} T\right) \exp \left\{\int_{n_{5} T}^{t}\left[a p_{r} \tilde{p}\left(S_{p}(\tau)-\varepsilon\right)-(\mu+d+\gamma)\right] \mathrm{d} \tau\right\} .
$$

It is easy to obtain that $\lim _{t \rightarrow \infty} I(t)=\infty$ from (28). This is a contradiction. Hence, the result (31) is true.

Finally, we will prove that

$$
\liminf _{t \rightarrow \infty} I(t) \geq \varepsilon
$$

for any solution $(S(t), I(t), R(t), U(t), V(t))$ of model (3) with initial value $X(0) \in \mathbb{R}_{+}^{5}$. By (28) we can chose two positive constants $\omega, \kappa$ such that

$$
\int_{t}^{t+\xi}\left[\operatorname{ap}_{r} \tilde{p}\left(S_{p}(\tau)-\varepsilon\right)-(\mu+d+\gamma)\right] \mathrm{d} \tau>\kappa
$$

for all $t \geq n_{5} T$ and $\xi>\omega$. If (40) were not true, then there would exist a sequence of initial values $Y_{n}=\left(S_{n}, I_{n}, R_{n}, U_{n}, V_{n}\right) \in \mathbb{R}_{+}^{5}(n=1,2, \ldots)$ such that

$$
\liminf _{t \rightarrow \infty} I\left(t, Y_{n}\right)<\frac{\varepsilon}{n^{2}}
$$

By (31), for every $n$, there are two time sequences $\left\{t_{j}^{(n)}\right\}$ and $\left\{s_{j}^{(n)}\right\}$ satisfying

$$
0<s_{1}^{(n)}<t_{1}^{(n)}<s_{2}^{(n)}<t_{2}^{(n)}<\cdots<s_{j}^{(n)}<t_{j}^{(n)}<\cdots
$$

and $\lim _{j \rightarrow \infty} s_{j}^{(n)}=\infty$ such that

$$
I\left(s_{j}^{(n)}, Y_{n}\right)=\frac{\varepsilon}{n}, \quad I\left(t_{j}^{(n)}, Y_{n}\right)=\frac{\varepsilon}{n^{2}},
$$

and

$$
\frac{\varepsilon}{n^{2}}<I\left(t, Y_{n}\right)<\frac{\varepsilon}{n} \quad \text { for all } t \in\left(s_{j}^{(n)}, t_{j}^{(n)}\right)
$$

From the second equation of model (3) we have

$$
\frac{\mathrm{d} I\left(t, Y_{n}\right)}{\mathrm{d} t}=a p_{r} S(t) V(t)-(\mu+d+\gamma) I(t) \geq-(\mu+d+\gamma) I\left(t, Y_{n}\right)
$$

Integrating this inequality from $s_{j}^{(n)}$ to $t_{j}^{(n)}$, it follows that

$$
I\left(t_{j}^{(n)}, Y_{n}\right) \geq I\left(s_{j}^{(n)}, Y_{n}\right) \exp \left\{-(\mu+d+\gamma)\left(t_{j}^{(n)}-s_{j}^{(n)}\right)\right\} .
$$


Therefore, by (42) we get

$$
\frac{\varepsilon}{n^{2}} \geq \frac{\varepsilon}{n} \exp \left\{-(\mu+d+\gamma)\left(t_{j}^{(n)}-s_{j}^{(n)}\right)\right\}
$$

that is,

$$
t_{j}^{(n)}-s_{j}^{(n)} \geq \frac{\ln n}{\mu+d+\gamma} \rightarrow \infty \quad \text { as } j \rightarrow \infty
$$

For each $n$, from the first equation of model (3) we have

$$
\frac{\mathrm{d} S(t)}{\mathrm{d} t} \geq \Lambda-a p_{r} \frac{\Lambda}{\mu}\left[\varepsilon_{1}+a p_{m}\left(M_{0}+\varepsilon\right) \varepsilon T\right]-\mu S(t) \quad \text { for all } t \in\left[s_{j}^{(n)}, t_{j}^{(n)}\right] .
$$

Let $\bar{S}(t)$ be a solution of model (34) with initial value $\bar{S}\left(s_{j}^{(n)}\right)=S\left(s_{j}^{(n)}, Y_{n}\right)$. By the comparison theorem of impulsive differential equation we get $S\left(t, Y_{n}\right) \geq \bar{S}(t)$. By (35) and (37) there is a constant $\widetilde{T}>0$, independent of $n$ and $j$, and there exists a positive constant $T^{*}$, independent of any $n$ and $j$, such that $t_{j}^{(n)}-s_{j}^{(n)}>T^{*}$. We chose $n$ large enough to make $t_{j}^{(n)}-s_{j}^{(n)}>T^{*}+\omega$. By (43) there exists $T_{1} \geq n_{5} T$ such that $F(\tilde{p}, t)=\tilde{p} I(t)-V(t) \leq 0$ for $t \geq T_{1}$.

Finally, from the second equation of model (3) and from (37) and (38) we have

$$
\frac{\mathrm{d} I(t)}{\mathrm{d} t}=a p_{r} S(t) V(t)-(\mu+d+\gamma) I(t) \geq\left[a p_{r} \tilde{p} S(t)-(\mu+d+\gamma)\right] I(t) .
$$

Integrating this inequality, from $s_{j}^{(n)}+T^{*}$ to $t_{j}^{(n)}$ follows that

$$
\begin{aligned}
\frac{\varepsilon}{n^{2}} & =I\left(t_{j}^{(n)}, Y_{n}\right) \geq I\left(s_{j}^{(n)}+T^{*}, Y_{n}\right) \\
& \geq \frac{\varepsilon}{n^{2}} \exp \left\{\int_{s_{j}^{(n)}+T^{*}}^{t_{j}^{(n)}}\left[a p_{r} \tilde{p}\left(S_{p}(\tau)-\varepsilon\right)-(\mu+d+\gamma)\right] \mathrm{d} \tau\right\} \\
& >\frac{\varepsilon}{n^{2}},
\end{aligned}
$$

where we uses (41). This leads to a contradiction, and so we finally proved that the (40) is true. From $F(\tilde{p}, t)=\tilde{p} I(t)-V(t) \leq 0$ for $t \geq T_{1}$ we have

$$
\liminf _{t \rightarrow \infty} V(t) \geq \liminf _{t \rightarrow \infty} \tilde{p} I(t) \geq \tilde{p} \varepsilon
$$

From this and from (40) we know that the RVFV disease in model (3) is uniformly persistent. This completes the proof of Theorem 3.

Using Corollary 1 given by Teng et al. in [30] on the existence of positive periodic solutions for the general impulsive ordinary differential equation, we have the following theorem on the existence of positive periodic solutions for periodic impulsive model (3).

Theorem 4 Suppose that the conditions of Theorem 3 hold. Then model (3) has at least one positive T-periodic solution. 
Table 1 Lists of parameters for Rift Valley Fever virus transmission

\begin{tabular}{|c|c|c|c|}
\hline Symbol & Description & Range & Source \\
\hline$\Lambda$ & Recruitment rate of ruminant & $25,000-46,000$ & Estimate \\
\hline$M_{0}$ & Capacity for mosquitoes & - & Estimate \\
\hline$a$ & Female mosquito bites rate $\left(\right.$ year $\left.^{-1}\right)$ & $156-256$ & Farida [18] \\
\hline$p_{m}$ & Probability of successful infection in mosquitoes & $(0.0021,0.2762)$ & Saul [10] \\
\hline$p_{r}$ & Probability of successful infection in ruminants & $(0.0021,0.2429)$ & Saul [10] \\
\hline d & Death rate of ruminant due to RVFV $\left(\right.$ year $^{-1}$ ) & $9.125-36.5$ & Saul [10] \\
\hline$\mu$ & Natural death rate of ruminant $\left(\right.$ year $^{-1}$ ) & $0.1014-1.0139$ & Saul [10] \\
\hline$\gamma$ & Rate of recovery in ruminant $\left(\right.$ year $^{-1}$ ) & $73-365$ & Saul [10] \\
\hline$\eta$ & Death rate of mosquitoes (year ${ }^{-1}$ ) & $6.08-121.6$ & Saul [10] \\
\hline$\xi$ & Development time of mosquitoes (year ${ }^{-1}$ ) & 24.3-73 & Saul [10] \\
\hline$\phi$ & Vaccinated rate for susceptible ruminant (year ${ }^{-1}$ ) & $0-1$ & Estimate \\
\hline
\end{tabular}

\section{Numerical simulation and discussion}

In this paper, we consider an epidemic model of RVFV with impulsive vaccination strategy. The main purpose is to investigate the impulsive vaccination thats governs whether the RVFV disease dies out or not and further to examine how the impulsive vaccination control strategy affects the prevention and control of RVFV disease. By using the comparison principle, integral and differential inequalities, and analytical methods, some sufficient conditions for the existence and stability of disease-free periodic solution and for uniform persistence of disease are obtained. Theoretical results show that RVFV disease can be controlled through changing the control parameters of model based on these conditions.

In this section, we perform some numerical simulations to illustrate the main theoretical results and the feasibility of impulsive vaccination control strategy for different control parameters using the Runge-Kutta method in the software MATLAB. The values of parameters for model (3) are listed in Table 1; we fixed the values of model parameters as follows: $\Lambda=32,000, a=256, p_{r}=7.8750 \times 10^{-3}, p_{m}=6.6406 \times 10^{-3}, \mu=0.12, d=12.0$, $\gamma=83.0$, and $\eta=95.0$.

We first choose the following control parameters: vaccination cycle $T=0.25$ (year) and immune strength $\phi=0.5$. It is easy to calculate that

$$
\begin{aligned}
\mathcal{R}_{0}= & \frac{1}{95 \times 0.25} \times\left[\frac{256 \times 7.8750 \times 10^{-3} \times 32,000}{0.12} \times 0.25\right. \\
& \left.+256 \times 6.6406 \times 10^{-3} \times 32,000 \times 0.25\right] \\
& +\frac{1}{95 \times 0.25} \times\left\{\frac{256 \times 7.8750 \times 10^{-3}}{0.12}\right. \\
& \left.\times\left[\frac{32,000 \times(1-0.5)\left(1-e^{-0.12 \times 0.25}\right)}{0.12 \times\left(1-(1-0.5) e^{-0.12 \times 0.25}\right)}-\frac{32,000}{0.12}\right] \times\left(1-e^{-0.12 \times 0.25}\right)\right\} \\
\approx & 0.9599<1
\end{aligned}
$$

by condition (10). Therefore, from Theorem 1 we know that model (3) has a disease-free periodic solution, which is globally asymptotically stable. The quantities of susceptible ruminants, infectious ruminants, and infectious mosquitoes of model (3) with impulsive vaccination or not are plotted against time in Figure 1(a)-(c) with blue lines and red lines, respectively. Further, the plot in Figure 1(d) shows the image of $\phi-T$ with $\mathcal{R}_{0}=1$, which 
(a)

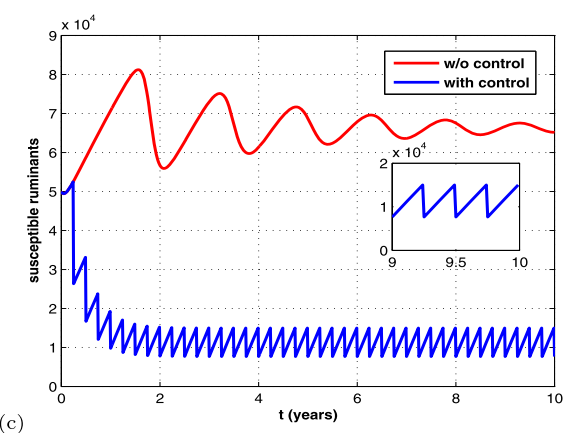

(c)

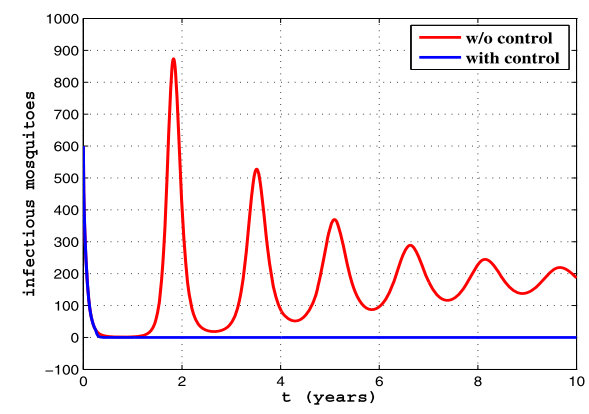

(b)

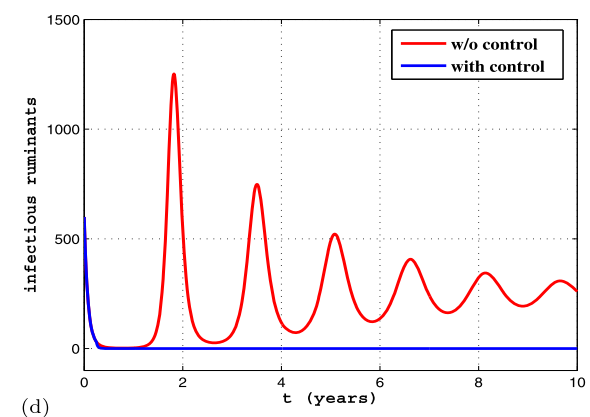

(d)

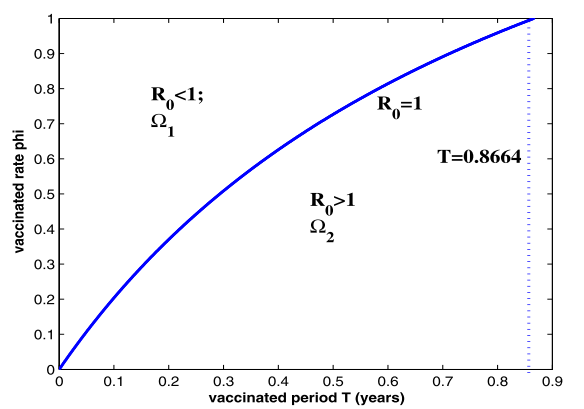

Figure 1 The stability of disease-free periodic solution of model (3) with $\mu=0.12, \gamma=83, d=12$, $p_{r}=0.00664 e^{-3}, p_{m}=0.00787 e^{-3}, \eta=95, \Lambda=32,000$, and control parameters $T=0.25, \phi=0.5$ : (a) susceptible ruminants $S(t)$; (b) infectious ruminants $/(t)$; (c) infectious mosquitoes $V(t)$; (d) the image of $\phi-T$ with $\mathcal{R}_{0}=1$.

indicates that model (3) has a disease-free periodic solution that is globally asymptotically stable in the region $\Omega_{1}$ for $\mathcal{R}_{0}<1$. This means that a short period of pulsing or a large pulse vaccination rate is a sufficient condition for the eradication of the RVFV. However, in the region $\Omega_{2}$ for $\mathcal{R}_{0}>1$, the dynamical behaviors of model (3) is complex, RVFV may or may not be extinct. These details are discussed in the next numerical simulations.

Next, we choose the control parameters $\phi=0.2$ and $T=0.8$ (year); the condition parameter $\tilde{p}$ of Theorem 3 is $\tilde{p}=0.6$, and others parameters are fixed as before. We compute that

$$
\begin{aligned}
\mathcal{R}_{1}= & {\left[\frac{256 \times 7.8750 \times 10^{-3} \times 0.6 \times 32,000}{0.12}-(0.12+12+83)\right] \times 0.8 } \\
& +\frac{256 \times 7.8750 \times 10^{-3} \times 0.6}{0.12} \\
& \times\left[\frac{32,000 \times(1-0.2) \times\left(1-e^{-0.12 \times 0.8}\right)}{0.12 \times\left(1-(1-0.2) \times e^{-0.12 \times 0.8}\right)}-\frac{32,000}{0.12}\right] \times\left(1-e^{-0.12 \times 0.8}\right) \\
\approx & 1.8475>0, \\
\mathcal{R}_{2}= & \left(\frac{1}{0.6} \times 256 \times 6,6406 \times 10^{-3} \times 0.8 \times 32,000-95\right) \times 0.8 \\
\approx & 14.6667,
\end{aligned}
$$




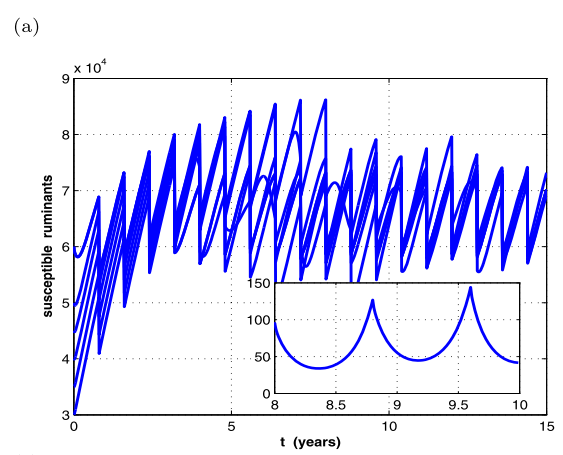

(c)

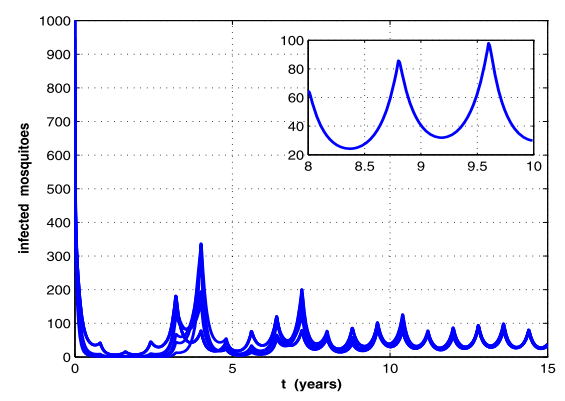

(b)

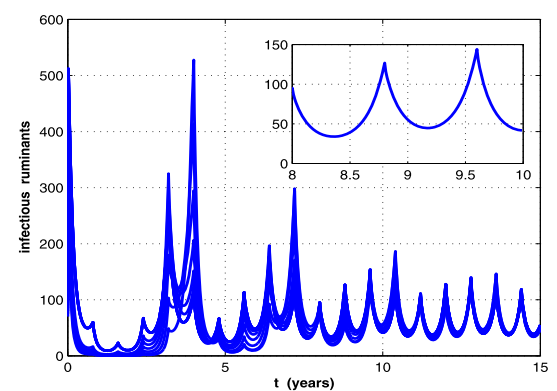

(d)

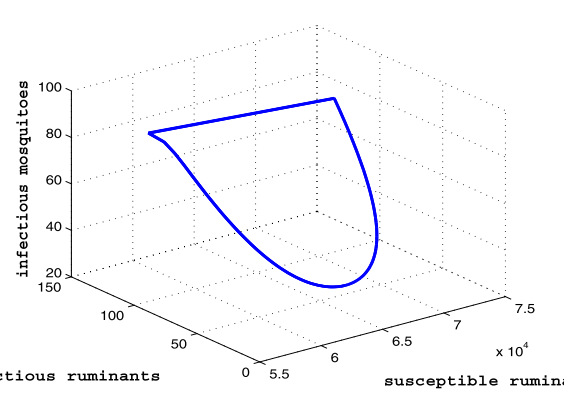

Figure 2 The uniform persistence of model (3) with $\mu=0.12, \gamma=83, d=12, p_{r}=0.00664 e^{-3}$, $p_{m}=0.00787 e^{-3}, \eta=95, \Lambda=32,000, T=0.2$, and $\phi=0.8$, where $\mathcal{R}=1.8475>0, \mathcal{R}^{*}=14.6667>0$, and $\boldsymbol{H}(\tilde{\boldsymbol{p}}, \boldsymbol{t})<0$ : (a) susceptible ruminants $S(t)$; (b) infectious ruminants $/(t)$; (c) infectious mosquitoes $V(t)$; (d) the image of susceptible ruminants $S(t)$, infectious ruminants $/(t)$ and infectious mosquitoes $V(t)$.

and

$$
\begin{aligned}
H(\tilde{p}, t)= & 256 \times 7.8750 \times 10^{-3} \times 0.6\left\{\frac{32,000}{0.12}\right. \\
& \left.+\left[\frac{32,000 \times(1-0.2) \times\left(1-e^{-0.12 \times 0.8}\right)}{0.12 \times\left(1-(1-0.2) \times e^{-0.12 \times 0.8}\right)}-\frac{32,000}{0.12}\right] \times e^{-0.12 \times(t-0.8)}\right\} \\
& +95-\frac{1}{0.6} \times 256 \times 6,6406 \times 10^{-3} \times 0.8 \times 32,000-(0.12+12+83) \\
\approx & -27.0032<0 .
\end{aligned}
$$

Obviously, RVFV is uniformly persistent, and model (3) has a positive periodic solution by Theorems 3 and 4. Figure 2(a)-(c) show that the numerical solutions of susceptible ruminants, infectious ruminants, and infectious mosquitoes with different initial values. Figure 2(d) shows the uniform persistence of infectious ruminants and infected mosquitoes. These simulations match the theoretical results very well. Theoretical results and numerical simulations show that RVFV is uniformly persistent if the immune strength $\phi$ is low and vaccination cycle $T$ is too long.

Finally, we consider the complex dynamical behaviors of model (3) when the condition in Theorem 1 hold and the conditions of Theorem 3 are invalid. Choosing the control parameters $\phi=0.2, T=0.8$, and $\tilde{p}=2$, we can calculate that $\mathcal{R}_{0} \approx 13.7132>1$, $\mathcal{R}_{1} \approx 183.7155>0, \mathcal{R}_{2} \approx 13.7600>0$, but $H(\tilde{p}, t) \approx 175.8473>0$. The quantities of infectious ruminants and infected mosquitoes are plotted in Figure 3(a) and (c); numerical 
(a)

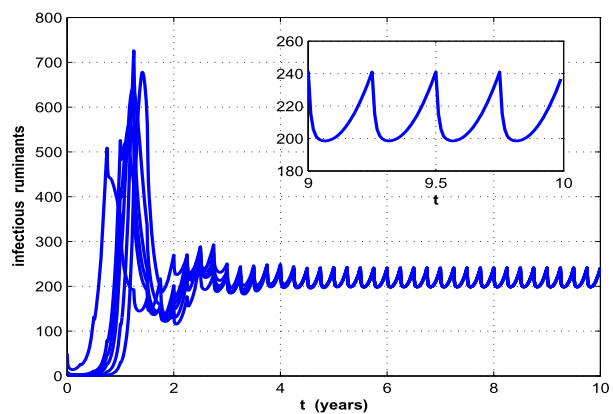

(c)

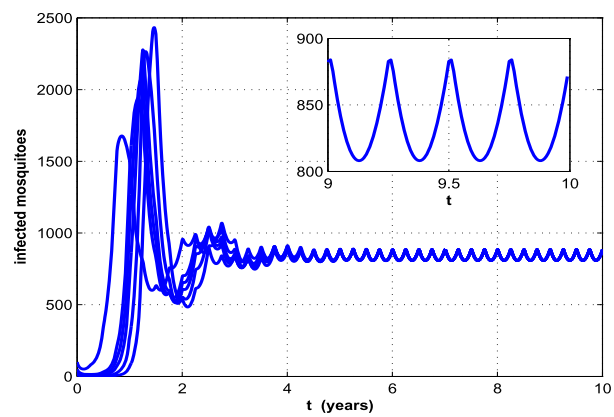

(b)
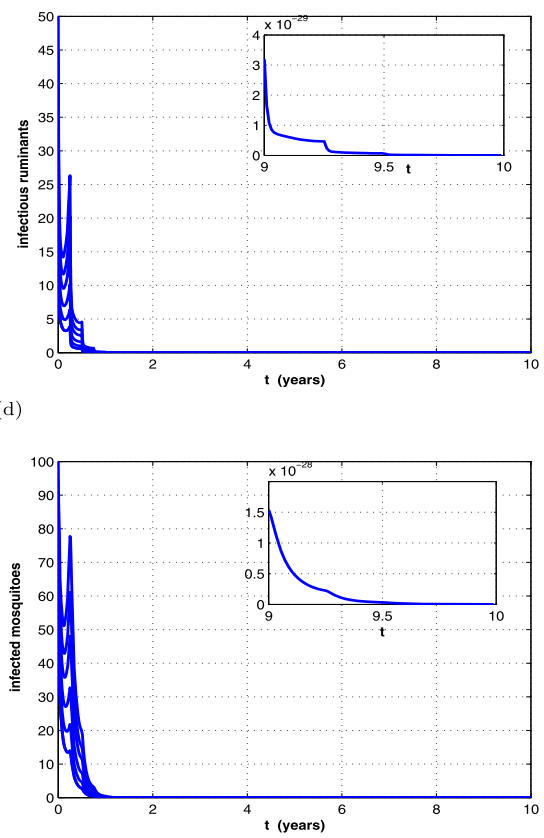

Figure 3 The complex dynamical behaviour of model (3) with $\mu=0.12, \gamma=83, d=12$, $p_{r}=0.00664 e^{-3}, p_{m}=0.00787 e^{-3}, \eta=95, \Lambda=32,000, T=0.8, \phi=0.2, \tilde{p}=2$, where the conditions of Theorem 3 are invalid: (a), (c) the uniform persistence of RVFV for $\mathcal{R}_{1}>0, \mathcal{R}_{2}>0$, and $H(\tilde{p}, t)>0(\phi=0.2$, $T=0.8)$; (b), (d) the extinction of RVFV for $\mathcal{R}_{1}<0, \mathcal{R}_{2}>0$, and $H(\tilde{p}, t)<0(\phi=0.8, T=0.5)$.

simulations show that RVFV is uniformly persistent. We choose, however, the control parameters $\phi=0.8, T=0.5$, and $\tilde{p}=2$, obtaining that $\mathcal{R}_{0} \approx 5.4344>1, \mathcal{R}_{1} \approx-24.2621<0$, $\mathcal{R}_{2} \approx 8.6000>0$, and $H(\tilde{p}, t) \approx-96.8909<0$. The plots in Figure $3(\mathrm{~b})$ and $(\mathrm{d})$ show that RVFV is died out. These show that the dynamical behaviors of model (3) are complex since the effects of impulsive vaccination strategy. These issues would be left as our future consideration.

\section{Competing interests}

The author declare that they have no competing interests.

\section{Authors' contributions}

The authors declare that the study was realized in collaboration with the same responsibility. All authors read and approved the final manuscript.

\section{Acknowledgements}

This research has been partially supported by the National Natural Science Foundation of China (Grant Nos. 11461067 11402223, and 11271312). The authors are very grateful to the editor and the anonymous referees for their valuable comments and suggestions, which greatly improved the presentation of this work.

Received: 26 November 2015 Accepted: 8 April 2016 Published online: 18 May 2016

\section{References}

1. Daubney, R, Hudson, JR, Granham, PC: Enzootic hepatitis or Rift Valley Fever: an underscribed virus disease of sheep cattle and man from East Africa. J. Pathol. Bacteriol. 34, 545-579 (1931)

2. Abdo-Salem, S, Tran, A, Grosbois, V, Gerbier, G, AL-Qadasi, M, Saeed, K, Etter, E, Thiry, E, Roger, F, Chevalier, V: Can environmental and socioeconomics factors explain the recent emergence of Rift Valley Fever in Yemen, 2000-2001? Vector-Borne Zoonotic Diseases 11, 773-779 (2011)

3. Mebus Charles, A: Rift Valley Fever. AccessScience. http://www.accessscience.com/content/rift-valley-fever/757367 (2014). Accessed 12 April 2016 
4. Anyambda, A, Chretien, JP, Small, J, Tucker, CJ, Formenty, PB, Richardson, JH, Britch, SC, Schnabelf, DC, Erickson, RL, Erickson, RL: Prediction of a Rift Valley fever outbreak. Proc. Natl. Acad. Sci. USA 106, 955-959 (2009)

5. Barnard, BJ: Rift Valley fever vaccine-antibody and immune response in cattle to a live and an inactivated vaccine. J. South Afr. Vet. Assoc. 50, 155-157 (1979)

6. Chitnis, N, Hyman, JM, Manore, CA: Modelling vertical transmission in vector-borne diseases with applications to Rift Valley fever. J. Biol. Dyn. 7, 11-40 (2013)

7. El-Rahim, IHA, Abd El-Hakim, U, Hussein, M: An epizootic of Rift Valley fever in Egypt in 1997. Rev. Sci. Tech. - Off. Int. Épizoot. 18(3), 741-748 (1999)

8. Xiao, YY, Beier, JC, Cantrell, RS, Cosner, C, DeAngelis, DL, Ruan, SG: Modelling the effects of seasonality and socioeconomic impact on the transmission of Rift Valley fever virus. PLoS Negl. Trop. Dis. 9, e3388 (2015)

9. Gaff, HD, Hartley, DM, Leahy, NP: An epidemiological model of Rift Valley fever. Electron. J. Differ. Equ. 2007, 115 (2007)

10. Mpeshe, SC, Haario, H, Tchuenche, JM: A mathematical model of Rift Valley fever with human host. Acta Biotheor. 59, 231-250 (2011)

11. Xue, L, Scott, HM, Cohnstaedt, LW, Scoglio, C: A network-based meta-population approach to model Rift Valley fever epidemics. J. Theor. Biol. 306, 129-144 (2012)

12. Gao, DZ, Cosner, C, Cantrell, RS, Beier, JC, Ruan, SG: Modeling the spatial spread of Rift Valley fever in Egypt. Bull. Math. Biol. 75, 523-542 (2013)

13. Al-Sulami, H, El-Shahed, M, Nieto, J, Shammmakh, W: On fractional order dengue epidemic model. Math. Probl. Eng. 2014, $456537(2014)$

14. Area, I, Batarfl, H, Losada, J, Nieto, J, Shammakh, W, Torres, A: On a fractional order Ebola epidemic model. Adv. Differ. Equ. 2015, 278 (2015)

15. Yang, HZ, Wei, HM, Li, XZ: Global stability of an epidemic model for vector-borne disease. J. Syst. Sci. Complex. 23, 279-292 (2010)

16. Davies, FG: Risk of a Rift Valley fever epidemic at the haj in Mecca, Saudi Arabia. Rev. Sci. Tech. - Off. Int. Épizoot. 25, 137-147 (2006)

17. Ikegami, T, Makinob, S: Rift Valley fever vaccines. Vaccine 27, 69-72 (2009)

18. Chamchod, F, Stephen, R, Cosner, C, Hassan, AN, Beie, JC, Ruan, SG: A modeling approach to investigate epizootic outbreaks and enzootic maintenance of Rift Valley fever virus. Bull. Math. Biol. 76, 2052-2072 (2014)

19. Sabin, AB: Measles: killer of millions in developing countries: strategies of elimination and continuing control. Eur. J. Epidemiol. 7, 1-22 (1991)

20. Ramsay, M, Gay, N, Miller, E: The epidemiology of measles in England and Wales: rationale for 1994 national vaccination campaign. Commun. Dis. Rep. 4(12), 141-146 (1994)

21. Shulgin, B, Stone, L, Agur, Z: Pulse vaccination strategy in the SIR epidemic model. Bull. Math. Biol. 60, 1123-1148 (1998)

22. d'Onofrio, A: Stability properties of pulse vaccination strategy in SEIR epidemic model. Math. Biosci. 179(1), 57-72 (2002)

23. Gao, SJ, Chen, LS, Nieto, JJ, Torres, A: Analysis of delayed epidemic model with pulse vaccination and saturation incidence. Vaccine 24, 6037-6045 (2006)

24. Gao, SJ, Ouyang, HS, Nieto, JJ: Mixed vaccination strategy in SIRS epidemic model with seasonal variability on infection. Int. J. Biomath. 4, 473-491 (2011)

25. Qiao, MH, Liu, AP, Foryś, U: Qualitative analysis of the SICR epidemic model with impulsive vaccinations. Math. Methods Appl. Sci. 36, 695-706 (2013)

26. Shulgin, B, Stone, L, Agur, Z: Pulse vaccination strategy in the SEIR model. Bull. Math. Biosci. 60(6), 1123-1148 (2000)

27. Zhang, $X B$, Xiang, H, Huo, HF, Meng, $X Y$, Xin, Y: An SIRS epidemic models with pulse vaccinations and non-monotonic incidence rate. Nonlinear Anal. Hybrid Syst. 8, 13-21 (2013)

28. Bainov, DD, Simeonov, PS: Impulsive Differential Equations: Periodic Solutions and Applications. Longman Scientific and Technical, New York (1993)

29. Lakshmikantham, V, Bainov, DD, Simeonov, PS: Theory of Impulsive Differential Equations. World Scientific, Singapore (1989)

30. Teng, ZD, Nie, LF, Fang, XN: The periodic solutions for general periodic impulsive population systems of functiona differential equations and its applications. Comput. Math. Appl. 61, 2690-2703 (2011)

\section{Submit your manuscript to a SpringerOpen ${ }^{\ominus}$ journal and benefit from:}

- Convenient online submission

Rigorous peer review

- Immediate publication on acceptance

- Open access: articles freely available online

- High visibility within the field

- Retaining the copyright to your article 